\title{
VTA GABA neurons modulate specific learning behaviors through the control of dopamine and cholinergic systems
}

\author{
Meaghan C. Creed, Niels R. Ntamati and Kelly R. Tan*
}

Department of Basic Neurosciences, University of Geneva, Geneva, Switzerland

\section{Edited by:}

Mary K. Lobo, University of Maryland

School of Medicine, USA

Reviewed by:

Joseph F. Cheer, University of

Maryland School of Medicine, USA

Ming-Hu Han, Mount Sinai School of

Medicine, USA

*Correspondence:

Kelly R. Tan, Department of Basic

Neurosciences, University of Geneva,

1 rue Michel Servet, CH 1211 Geneva,

Switzerland

e-mail: kelly.tan@unige.ch
The mesolimbic reward system is primarily comprised of the ventral tegmental area (VTA) and the nucleus accumbens (NAc) as well as their afferent and efferent connections. This circuitry is essential for learning about stimuli associated with motivationally-relevant outcomes. Moreover, addictive drugs affect and remodel this system, which may underlie their addictive properties. In addition to dopamine (DA) neurons, the VTA also contains approximately 30\% $\gamma$-aminobutyric acid (GABA) neurons. The task of signaling both rewarding and aversive events from the VTA to the NAc has mostly been ascribed to DA neurons and the role of GABA neurons has been largely neglected until recently. GABA neurons provide local inhibition of DA neurons and also long-range inhibition of projection regions, including the NAc. Here we review studies using a combination of in vivo and ex vivo electrophysiology, pharmacogenetic and optogenetic manipulations that have characterized the functional neuroanatomy of inhibitory circuits in the mesolimbic system, and describe how GABA neurons of the VTA regulate reward and aversion-related learning. We also discuss pharmacogenetic manipulation of this system with benzodiazepines (BDZs), a class of addictive drugs, which act directly on $\mathrm{GABA}_{A}$ receptors located on GABA neurons of the VTA. The results gathered with each of these approaches suggest that VTA GABA neurons bi-directionally modulate activity of local DA neurons, underlying reward or aversion at the behavioral level. Conversely, long-range GABA projections from the VTA to the NAc selectively target cholinergic interneurons (CINs) to pause their firing and temporarily reduce cholinergic tone in the NAc, which modulates associative learning. Further characterization of inhibitory circuit function within and beyond the VTA is needed in order to fully understand the function of the mesolimbic system under normal and pathological conditions.

Keywords: benzodiazepine, dopamine, acetylcholine, NAc, VTA, interneuron, pharmacogenetics, optogenetics

\section{INTRODUCTION}

Learning about motivationally relevant stimuli in the environment is critical for all aspects of survival, from feeding and reproduction to avoiding dangerous or aversive situations. This learning is primarily mediated by the mesolimbic system. Often referred to as the reward system, the mesolimbic system is primarily comprised of the ventral tegmental area (VTA) and the nucleus accumbens (NAc) as well as their afferent and efferent connections (Figure 1). Signaling the salience of an event or stimulus from the VTA in the midbrain to the NAc and other forebrain regions has largely been ascribed to dopamine (DA) neurons (Berridge and Robinson, 1998; Smith et al., 2011). Over the past several decades, extensive studies at the cellular and behavioral levels have shown that populations of DA neurons in the VTA increase their behavior in response to rewarding stimuli (Schultz et al., 1993). In response to aversive stimuli, the activity of most DA neurons is silenced (Mileykovskiy and Morales, 2011; Cohen et al., 2012), although a subset of DA neurons show activation (Joshua et al., 2008). Over training with reward experience, this DA activation will occur not only in response to the salient primary stimuli per se, but will also occur in response to previously neutral cues in the environment that the animal has associated with these motivationally relevant outcomes (Schultz, 2013). As a consequence of DA activity, DA in the NAc induces a motivational drive, and this DA signal is modulated by past experience of reward and punishment (Oleson et al., 2012; Howe et al., 2013). In this way, mesolimbic DA has been conceptualized as a teaching signal, coding the magnitude of aversive and rewarding environmental stimuli and increasing behavioral vigor related to these salient stimuli (Peciña and Berridge, 2013). The rewarding effect of addictive drugs is also mediated by mesolimbic DA. Following exposure to drugs of abuse, DA is elevated throughout the mesolimbic system (Lüscher and Ungless, 2006). This persistent increase in DA results in pathological salience attributed to drug-associated cues, and compulsive drug use despite negative consequences. In this way, drug addiction is an example of adverse behavioral consequences arising from a malfunction of the mesolimbic system.

As previously discussed, the role of DA in the mesolimbic system has been the subject of intense investigation over the 


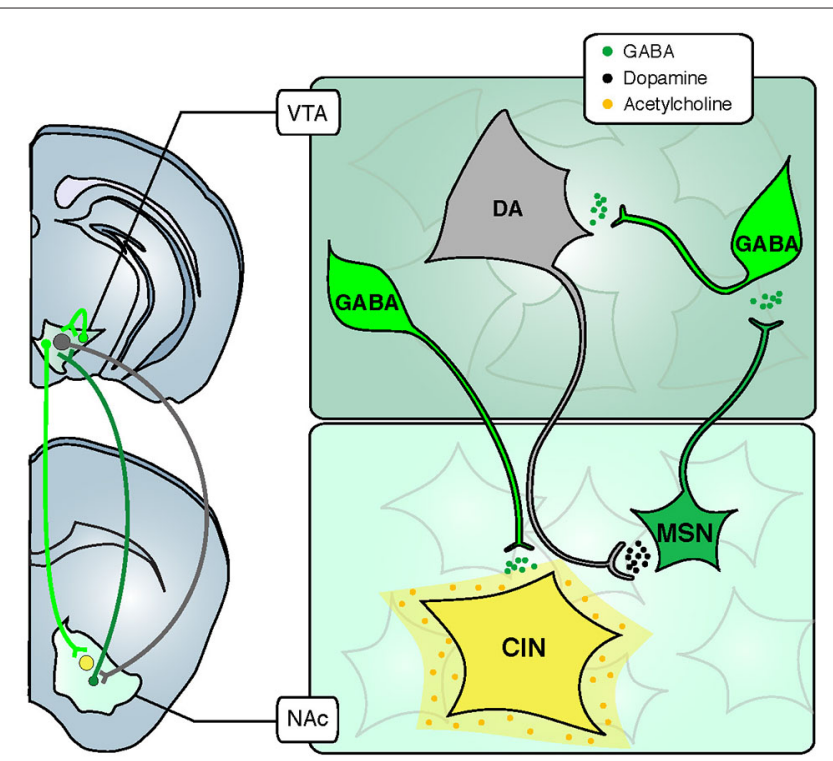

FIGURE 1 | Inhibitory and modulatory connections between the VTA and the NAc. The VTA is composed of two major cell types: GABAergic neurons (green) and DA neurons (grey). Glutamate neurons are not schematized for clarity. GABA interneurons control the activity of DA cells that release DA in the NAc through their long-range projections. In turn, MSNs send projections back to the VTA, preferentially inhibiting GABA cells and thus disinhibiting DA neurons. Some GABA neurons also send long-range projections to the NAc, where they selectively inhibit CINs. Abbreviations: VTA: ventral tegmental area; NAc: nucleus accumbens; GABA: $\gamma$-aminobutyric acid; DA: dopamine; CIN: cholinergic interneuron; MSN: medium sized-spiny neuron.

past several decades. However, in addition to DA, the VTA also contains approximately 30\% $\gamma$-aminobutyric acid (GABA) neurons, and the role of these inhibitory neurons in the mesolimbic system is less well understood. Within the VTA, there are two general populations of GABA neurons: interneurons, which provide local inhibition of DA neurons and projection neurons, which provide long-range inhibition of multiple brain areas including the NAc (Figure 1). In general, inhibition is critical for regulating neuronal excitability, and allows flexibility in circuit connectivity. A consequence of this flexibility is plasticity within the mesolimbic system, which permits reward-related learning. Despite their likely functional consequences, the role of VTA GABA neurons in reward- and aversion-related learning is not well understood. Until recently, it has been difficult to disentangle the function of DA neurons and GABA neurons, and between local VTA GABA neurons and GABAergic projection neurons (GPNs).

In this review we discuss studies using optogenetic and pharmacogenetic tools to dissect the precise role of GABAergic neurons in the mesolimbic system. Much of this recent work has characterized the functional neuroanatomy of inhibitory circuits in the mesolimbic system, and has begun to elucidate the precise mechanisms by which these inhibitory circuits regulate activity of DA neurons and other neuromodulatory systems, such as acetylcholine (ACh). It is now evident that these inhibitory circuits critically regulate DA neuron function and reward-related learning. VTA GABA neurons bi-directionally modulate activity of local DA neurons, which underlies reward or aversion at the behavioral level. Conversely, long-range GABA projections from the VTA to the NAc selectively target cholinergic interneurons (CINs; Figure 1), regulating local ACh release and modulating associative learning. We also use the example of drug addiction to discuss how inhibitory neurons contribute to dysfunction of the mesolimbic system and consequent maladaptive behaviors. Continuing to clarify the role of inhibitory neurons within the VTA and beyond will be necessary to fully understand the function of the mesolimbic system under normal and pathological conditions.

\section{ACTIVATION OF LOCAL GABA NEURONS IN THE VENTRAL TEGMENTAL AREA (VTA)}

As mentioned, the VTA contains a large proportion of GABA neurons ( $230 \%$; Dobi et al., 2010). We now appreciate the intimate role these neurons play in regulating activity of DA neurons, and the behavioral consequences of their activity. GABA released from local VTA neurons profoundly affects the activity of VTA DA neurons. Using Cre mice (GAD-cre (Tan et al., 2012) or VGAT-cre (van Zessen et al., 2012) to express channelrhodopsin 2 (ChR2) selectively in GABAergic neurons), it has been possible to selectively manipulate VTA GABA neurons in vitro and in vivo, and determine the effects on DA neurons as well as the consequences of this activation on behavior (Figure 2). Using in vivo extracellular recording in VTA ChR2 injected and anesthetized GAD-cre mice to monitor DA neuron activity, we have shown that driving activity of VTA GABA neurons strongly inhibits DA neuron spontaneous firing rate (Tan et al., 2012; Figure 2A). In stark contrast, shutting down the activity of GABA neurons, by expressing the proton pump halorhodpsin in VTA GABA neurons, leads to an increase or disinhibition of DA cells (Bocklisch et al., 2013). These results were confirmed with ex vivo patch clamp electrophysiology, showing that local VTA GABA neurons make direct synaptic connections with DA neurons. Blue-light activation of ChR2-expressing GABA neurons induced inhibitory post-synaptic responses in neighboring DA neurons. These lightevoked currents were abolished by the sodium channel blocker tetrodotoxin or by the chloride channel blocker picrotoxin, confirming a monosynaptic connection mediated by $\mathrm{GABA}_{\mathrm{A}}$ receptors (Tan et al., 2012; van Zessen et al., 2012). Through activation of $\mathrm{GABA}_{\mathrm{A}}$ receptors, local GABA cells control their target DA neurons, decreasing their excitability, thereby balancing excitation from glutamatergic inputs (Overton and Clark, 1992).

This inhibitory control has important consequences for DA function and downstream behavior, particularly regarding significance to motivationally relevant outcomes (Schultz et al., 1997; Schultz, 2006; Fields et al., 2007; Bromberg-Martin et al., 2010). Specifically, exposure to salient but aversive stimuli can lead to the inhibition of VTA DA neurons (Mirenowicz and Schultz, 1996; Ungless et al., 2004, 2010; Brischoux et al., 2009; Matsumoto and Hikosaka, 2009; Hong et al., 2011; Zweifel et al., 2011; Tan et al., 2012; van Zessen et al., 2012). Recordings in anesthetized animals have demonstrated that aversive stimuli (such as a footshock) transiently and potently increase the spontaneous firing rate of GABAergic neurons of the VTA and decrease the activity of DA neurons (Ungless et al., 2004; Hong et al., 2011; Tan et al., 


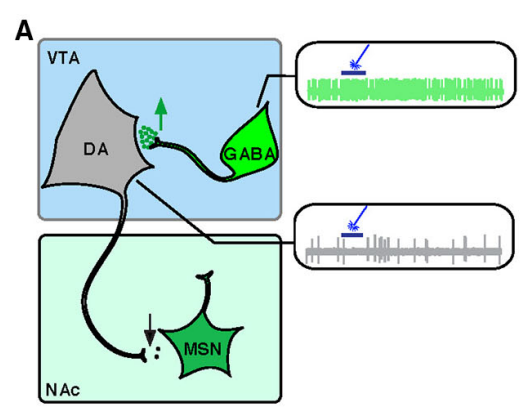

\section{Dynamic Conditioned Place Aversion}
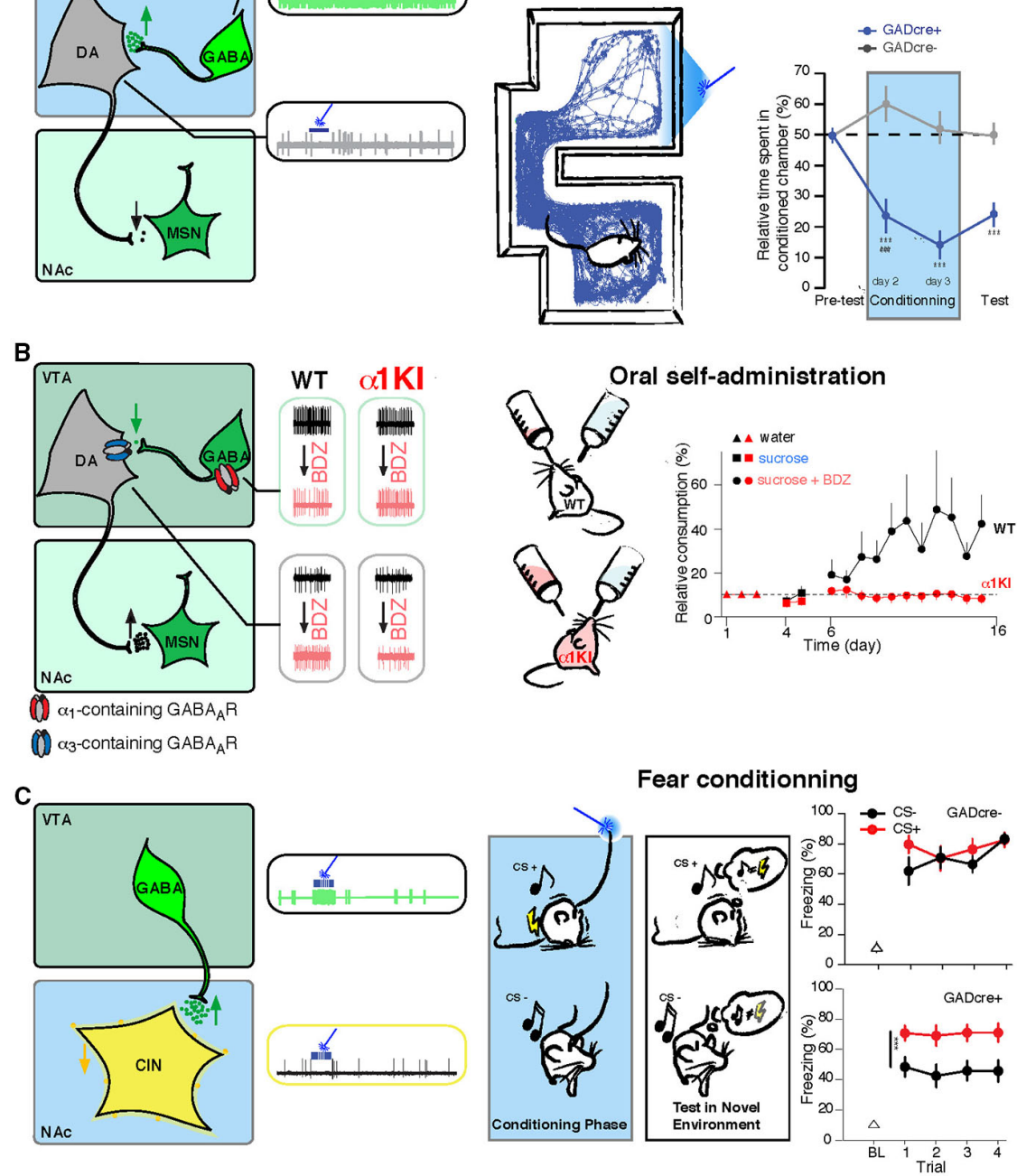

FIGURE 2 | VTA GABA neurons drive specific behaviors through the control of the DA and cholinergic systems. (A) Left, VTA GABA neurons are selectively infected to express ChR2-eYFP (green, AAV-ChR2-eYFP-flox in the VTA of GADcre+ mice). Middle, in vivo example trace of single unit recording of a GABA and a DA cell showing that blue-light stimulation induces a time locked increase of firing rate in infected GABA neurons and as a consequence a shut down in the activity of DA cells. Right, dynamic conditioned place aversion (CPA): GADcre+ mice developed an aversion for the blue-light paired chamber compared to GADcre- mice. This is shown on the example tracking trace of a GADcre+ mouse and on the aversive learning curve plotting the relative time spent in the conditioned chamber for both groups of mice. (B) Left, the ventral tegmental area (VTA) contains DA neurons expressing $\alpha 3$-containing $\mathrm{GABA}_{A}$ receptors and GABA cells that express $\alpha 1$-containing $\mathrm{GABA}_{A}$ receptors. Middle, in vivo example trace of single unit recording of GABA and DA cells before and after midazolam (MDZ) tail vein injection. GABA neurons decrease their basal firing rate, leading to the disinhibiton of DA cells, which increase their firing rate. These effects are abolished in $\alpha 1$-knock-in mice in which $\mathrm{GABA}_{\mathrm{A}}$ receptors containing the $\alpha 1$ subunit isoform are insensitive to BDZs. Right, oral-self administration of MDZ paradigm: WT mice preferred to consume from the MDZ containing solution whereas this preference is not developed in $\alpha 1$-knock-in mice. (C) Left, same preparation as in B, VTA GABA projection neurons selectively inhibit CIN of the NAc. Middle, in vivo example trace of single unit recording of GABA and CIN cells showing that in response to blue-light stimulation CIN are completely inhibited due to the excitation of VTA GABA cells. Right, activation of these fibers during the association of a conditioned stimulus (CS+, white noise) to an aversive outcome (brief mild footshock) enhances the ability to subsequently discriminate between the conditioned stimulus and the unconditioned stimulus (CS, pure tone) that was not paired with an aversive outcome, as shown on the graph for the percentage of freezing measured on the test day in response to $\mathrm{CS}+$ and $\mathrm{CS}-$ for both groups of mice. Abbreviations: VTA: ventral tegmental area; NAc: nucleus accumbens; WT: wild type mouse; $\alpha 1 \mathrm{KI}$ : $\alpha 1$ subunit point mutant knock-in mouse; BDZ: benzodiazepine; GABA: $\gamma$-aminobutyric acid; DA: dopamine; CIN: cholinergic interneuron; MSN: medium sized-spiny neuron. Adapted from Tan et al. (2010, 2012) and Brown et al. (2012).
2012). Likewise, in vivo recording in awake behaving animals determined that aversive stimuli lead to excitation of the majority of VTA GABA neurons, which were optogenetically identified (Cohen et al., 2012). Conversely, mixed responses were observed in DA neurons, with the predominant response being inhibition (Brischoux et al., 2009; Cohen et al., 2012; Tan et al., 2012). The increase in GABA neuron spontaneous firing rate and decrease in DA neuron activity following the aversive stimuli of a foot-shock 
were prevented by a $\mathrm{GABA}_{\mathrm{A}}$ receptor antagonist, but were not affected by DA antagonists (Tan et al., 2012), indicating that the effects on DA firing rate were downstream of the increased activity of GABA neurons.

Confirming the regulatory role of VTA GABA neurons on motivationally relevant behaviors, driving local VTA GABA neurons with ChR2 results in strong aversion to a compartment associated with light stimulation (Tan et al., 2012). In this dynamic conditioned place aversion (CPA) paradigm (also named real time CPA task), the VTA of ChR2-infected GAD-cre mice was stimulated with blue light when the animal was exploring one of the two compartments, and the light was turned off as soon as the mice exited this chamber. On the test day, where no blue light is applied to the VTA, an aversion of the light paired-chamber was measured (Figure 2A). Interestingly, the CPA produced in response to activating VTA GABA neurons persisted during test sessions, indicating a strong learning effect resulting from DA neuron inhibition. Furthermore, driving VTA GABA neurons also disrupted reward consumption, as measured in a free-sucrose access paradigm (van Zessen et al., 2012). In another study, in vivo recordings of the activity of VTA cells were done while mice learned to associate an odor with appetitive rewarding or aversive outcomes. GABA neurons increased their firing during the delay between a conditioned stimulus (odor) and an unconditioned stimulus (reward or aversive air puff), while the firing rate was not changed during the delivery of the reward or punishment. The authors speculated that this ramping up of GABAergic firing may modulate DA activity during reward expectation and may help compute the reward prediction error (Cohen et al., 2012). Such cellular mechanism of action has been correlated with subsecond DA release measurements in the NAc of rat undergoing fearconditioning tasks. These studies showed that a warning signal presentation (Oleson et al., 2012; Olesson and Cheer, 2013) or a fear conditioning cue (Badrinarayan et al., 2012) result in decreased DA levels as measured by fast scan cyclic voltametry.

Taken together, these observations implicate VTA GABA neuron activity in learning motivationally relevant outcomes associated with unconditioned stimuli. Specifically, driving VTA GABA neurons leads to negative modulation of DA neurons and aversion at the behavioral level. This learning signal may have implications for the involvement of VTA GABA neurons in maladaptive learned behaviors, such as those that characterized in drug addiction.

\section{INHIBITION OF LOCAL GABA NEURONS IN THE VENTRAL TEGMENTAL AREA (VTA)}

VTA GABA neurons receive inhibitory inputs mainly from medium sized-spiny neuron (MSN) of the NAc (Xia et al., 2011). Activation of MSNs with ChR2, for instance, leads to the inhibition of VTA GABA cells, and consequent disinhibition of DA neurons (Bocklisch et al., 2013). This mechanism of cellular disinhibition is implicated in the rewarding effects of several classes of addictive drugs (Lüscher and Ungless, 2006). Specifically, opioids (Johnson and North, 1992a), gamma-hydroxy butyric acid (GHB) (Cruz et al., 2004), cannabinoids (Oleson and Cheer, 2012) and benzodiazepines (BDZs; Tan et al., 2010, 2011) act on VTA GABA neurons (Johnson and North, 1992b) and inhibit their activity.
We and others have examined how decreasing the activity of VTA GABA neurons, contributes to the synaptic and behavioral adaptations induced by addictive drugs.

BDZs are a class of drug used in clinic to treat anxiety, insomnia and muscle spasms. They also induce sedation and anterograde amnesia and unfortunately, they carry the potential for addiction. All these effects are mediated through BDZs action as positive allosteric modulators of $\mathrm{GABA}_{\mathrm{A}}$ receptors; they potentiate inhibition in the brain. In vivo administration of BDZs inhibits the activity of VTA GABA neurons and consequently increases DA neuron's firing rate (Figure 2B). Along with BDZs, opioids are another class of addictive drugs that act initially on GABA neurons through binding to $\mu$-opioid receptors specifically expressed on GABA cells (Pickel et al., 2002), decreasing their activity thus leading to the disinhibition of VTA DA neurons (Johnson and North, 1992a).

All addictive drugs, such as cocaine, amphetamine, morphine, $\mathrm{GHB}$, nicotine and ethanol induce characteristic forms of synaptic plasticity on VTA DA neurons within $24 \mathrm{~h}$ following an acute exposure (Lüscher and Malenka, 2011). Specifically, an increase in excitatory synaptic strength (as measured by the ratio of the amplitude of AMPA to NMDA-mediated currents (Ungless et al., 2001)) is observed, as well as inward rectification of the AMPA current at positive potentials (Bellone and Lüscher, 2006), which results from the insertion of calcium-permeable AMPA receptors. Interestingly, activation of DA neurons is sufficient to induce this plasticity, since optogenetic activation of these neurons alone (using DA transporter-cre mice) induces the same form of plasticity (Brown et al., 2010). BDZs also trigger this critical feature (Heikkinen et al., 2009; Tan et al., 2010) indicating that they share a common mechanism of DA regulation with other classes of addictive drugs.

Using a pharmacogenetic approach, we demonstrated that the effect of BDZs and more specifically midazolam (MDZ, a BDZ potentiating all $\mathrm{GABA}_{\mathrm{A}}$ receptors) on neuronal activity and plasticity are mediated by $\alpha 1$-containing $\mathrm{GABA}_{\mathrm{A}}$ receptors. In the $\alpha 1(\mathrm{H} 101 \mathrm{R})$ point mutant mouse line, which carries a single nucleotide mutation that disrupts binding of BDZs to the $\mathrm{GABA}_{\mathrm{A}} \mathrm{R}$ binding site (Rudolph et al., 1999), all effects of MDZ are abolished (Tan et al., 2010). Moreover, unlike wildtype mice, these $\alpha 1(\mathrm{H} 101 \mathrm{R})$ mice did not self-administer MDZ in an oral-self administration task where they had the option to drink from two bottles, differing only in that one contains a MDZ (Figure 2B).

Inhibition of VTA GABA cells results in the release of the inhibitory break onto DA neurons. Such disinhibition represents a means to trigger the release of DA in target brain regions to modulate synaptic transmission and hence regulate specific behaviors such as the oral-self administration of BDZs, a rewardrelated behavior.

\section{LONG-RANGE GABAERGIC PROJECTIONS FROM THE VENTRAL TEGMENTAL AREA (VTA)}

VTA DA neurons are long-range projection neurons, which target the prefrontal cortex and the NAc, among other areas. These same areas are also innervated by a subset of VTA GPNs; these neurons comprise approximately $25 \%$ of VTA GABA cells (Margolis et al., 
2006; Figure 1). In the NAc, GPNs target CINs nearly exclusively, with very sparse innervation of MSNs (Brown et al., 2012). Conversely, VTA DA neurons modulate both MSNs and CINs, either through volume transmission or direct synaptic contacts (Moss and Bolam, 2008).

Using ex vivo whole cell recording methods in GAD-cre mice expressing ChR2 in VTA GABA cells, we showed that the connection between VTA GPNs and CINs in the NAc is monosynaptic. Light-evoked currents were recorded in $100 \%$ of CINs and blocked by picrotoxin or tetrodotoxin, whereas such currents were rarely detected in MSNs or parvalbumin-expressing interneurons (Brown et al., 2012). In vivo recordings confirmed that activating VTA GABA neurons at physiological frequencies potently inhibited CINs for the duration of the stimulation (Figure 2C). This anatomical and functional connectivity has important consequences for associative learning. In a fear conditioning task, when GPNs were optogenetically activated during presentation of a specific tone paired with a foot-shock, mice showed increased ability to discriminate this conditioned tone from an unconditioned tone (Brown et al., 2012; Figure 2C).

During Pavlovian conditioning, VTA DA neurons increase their firing in response to a stimulus predicting either reward or aversion resulting in increased DA release in the NAc. In parallel, the inhibition of CINs produced by GPNs would modulate information processing by MSNs either directly through the actions of muscarinic receptors (Surmeier et al., 2007) or by increasing DA release from VTA terminal fields in the NAc (Cragg and Rice, 2004). Moreover, CINs express DA D2 receptors and are subject to DA regulation (Alcantara et al., 2003) and tonic activity of CINs elicits DA release (Cachope et al., 2012). In this way, through their actions on MSNs and CINs, VTA GABA and DA neurons interact to coordinate activity and reward processing of the NAc. Interestingly, however, inhibiting GPNs terminating in the NAc was insufficient to disrupt reward consumption. These observations support a dual mechanism of VTA to NAc communication, in which targeting of NAc CINs by VTA GPNs and of MSNs and CINs by DA neurons operates in concert to enable the association of motivationally relevant outcomes to unconditioned stimuli.

\section{CONCLUDING REMARKS}

Within the mesolimbic reward system, communication of rewarding and aversive stimuli has largely been ascribed to DA neurons. The firing rate of DA neurons responds to salient stimuli that are both rewarding and aversive in valence, and is involved in computing reward prediction error. These DA neurons project to regions throughout the brain, including the NAc, which is implicated in behavioral responses to rewarding and aversive stimuli. As such, the mesolimbic DA system is critically involved in learning about motivationally-relevant outcomes. However, in addition to DA cells, the VTA contains GABA neurons, which synapse locally onto DA neurons, and send long-range projections to brain areas innervated by VTA DA neurons. Until recently, the role of VTA GABA neurons on the regulation of DA neurons and on reward-learning was not well understood. However, with the advent of selective optogenetic and pharmacogenetic tools, the role of these inhibitory neurons within the mesolimbic reward system has begun to be elucidated.
We have discussed how within the VTA, aversive stimuli drive the activity of GABA neurons, leading to inhibition of DA neuron firing. Conversely, inhibiting the activity of these VTA GABA neurons (for example, by administration of BDZs) increases the activity of DA neurons and is behaviorally rewarding. The contribution of long-range GABA projection neurons to mesolimbic system function, and the interaction of GPNs with DA projection neurons at the level of their projection areas have also begun to be investigated. For example, despite the established role of the NAc in the maintenance of reward-related behavior (From Introduction), optical inhibition of GPN afferents in the NAc had no effect on reward consumption (van Zessen et al., 2012), and produces no aversive state per se (Brown et al., 2012). By confirming that disruption of GABAergic signaling from VTA GPNs to the NAc is insufficient to disrupt reward or produce aversion, these observations further underscore the critical role of DA projections in reward-related learning and coding the valence of environmental stimuli. However, driving activity of GPNs from the VTA to NAc enhances associative learning despite this lack of effect on consumatory behavior or aversion. These observations lead us to the question of the heterogeneity of VTA GABA cells. Within the NAc, antidromic optical activation of VTA GABA neurons inhibited approximately $25 \%$ of VTA DA neurons (Brown et al., 2012), suggesting that only a subset of VTA GABA neurons project to the NAc and some others have local collaterals. In addition the population of GABA interneurons that do not project outside the VTA, it is likely that a distinct set of GABA neurons project to other brain regions involved in reward processing and behavior, such as the PFC and hippocampus.

To fully appreciate the role of inhibition in the mesolimbic system, a better understanding of upstream inputs onto VTA GABA neurons, and the role of GABA neurons originating in other nodes of the mesolimbic system are needed. Optogenetic manipulations have recently been used to explore both of these issues. Using these tools to map functional connectivity, it was revealed that both bed-nucleus of the stria terminalis (BNST) glutamatergic and GABAergic projections preferentially innervate putative GABA neurons in the VTA (Jennings et al., 2013). In vivo photostimulation of BNST glutamatergic projections resulted in aversive and anxiogenic behavioral phenotypes. Conversely, activation of BNST GABAergic projections produced rewarding and anxiolytic phenotypes, similarly to direct activation of VTA GABA cells (Jennings et al., 2013). Another source of input onto VTA GABA cells is through back-projections from the NAc. Dopamine receptor type 1 (D1R)-receptor-expressing MSNs, but not Dopamine receptor type 2 (D2R)-expressing MSNs of the NAc form functional synaptic contacts with GABA neurons in the VTA (Xia et al., 2011; Bocklisch et al., 2013). This connection is potentiated following exposure to cocaine (Bocklisch et al., 2013), and inhibitory transmission from VTA back to the NAc is suppressed (Ishikawa et al., 2013). These studies provide crucial information about upstream inputs onto VTA GABA neurons and may provide further insight into how DA release is controlled under normal and pathological conditions.

DA modulation is important for function of reward, aversion and reward related learning. While much work has been devoted to studying the function of DA neurons and their role in encoding 
salient information of rewarding or aversive in nature, the role of VTA GABA neurons has been less well characterized. However, as we have discussed, it is now understood that GABA neurons regulate the activity of both VTA DA neurons and target neurons in VTA projection areas. This intimate, bi-directional modulation of DA neurons not only contributes to reward prediction error and DA plasticity, but also encodes the valence state of rewarding and aversive stimuli and also helps orchestrate behavioral responses to salient stimuli. Further characterization of inhibitory circuit function within and beyond the VTA will be necessary to fully understand the function of the mesolimbic system under normal and pathological conditions.

\section{REFERENCES}

Alcantara, A. A., Chen, V., Herring, B. E., Mendenhall, J. M., and Berlanga, M. L. (2003). Localization of dopamine D2 receptors on cholinergic interneurons of the dorsal striatum and nucleus accumbens of the rat. Brain Res. 986, 22-29. doi: 10.1016/s0006-8993(03)03165-2

Badrinarayan, A., Wescott, S. A., Vander Weele, C. M., Saunders, B. T., Couturier, B. E., Maren, S., et al. (2012). Aversive stimuli differentially modulate realtime dopamine transmission dynamics within the nucleus accumbens core and shell. J. Neurosci. 32, 15779-15790. doi: 10.1523/JNEUROSCI.3557-12. 2012

Bellone, C., and Lüscher, C. (2006). Cocaine triggered AMPA receptor redistribution is reversed in vivo by mGluR-dependent long-term depression. Nat. Neurosci. 9, 636-641. doi: 10.1038/nn1682

Berridge, K. C., and Robinson, T. E. (1998). What is the role of dopamine in reward: hedonic impact, reward learning, or incentive salience? Brain Res. Brain Res. Rev. 28, 309-369. doi: 10.1016/s0165-0173(98)00019-8

Bocklisch, C., Pascoli, V., Wong, J. C. Y., House, D. R. C., Yvon, C., de Roo, M., et al. (2013). Cocaine disinhibits dopamine neurons by potentiation of GABA transmission in the ventral tegmental area. Science 341, 1521-1525. doi: 10. 1126/science.1237059

Brischoux, F., Chakraborty, S., Brierley, D. I., and Ungless, M. A. (2009). Phasic excitation of dopamine neurons in ventral VTA by noxious stimuli. Proc. Natl. Acad. Sci. U S A 106, 4894-4899. doi: 10.1073/pnas.0811507106

Bromberg-Martin, E. S., Matsumoto, M., and Hikosaka, O. (2010). Dopamine in motivational control: rewarding, aversive, and alerting. Neuron 68, 815-834. doi: 10.1016/j.neuron.2010.11.022

Brown, M. T. C., Bellone, C., Mameli, M., Labouèbe, G., Bocklisch, C., Balland, B., et al. (2010). Drug-driven AMPA receptor redistribution mimicked by selective dopamine neuron stimulation. PLoS One 5:e15870. doi: 10.1371/journal.pone. 0015870

Brown, M. T. C., Tan, K. R., O’Connor, E. C., Nikonenko, I., Muller, D., and Lüscher, C. (2012). Ventral tegmental area GABA projections pause accumbal cholinergic interneurons to enhance associative learning. Nature 492, 452-456. doi: 10.1038/nature11657

Cachope, R., Mateo, Y., Mathur, B. N., Irving, J., Wang, H.-L., Morales, M., et al. (2012). Selective activation of cholinergic interneurons enhances accumbal phasic dopamine release: setting the tone for reward processing. Cell Rep. 2, 3341. doi: 10.1016/j.celrep.2012.05.011

Cohen, J. Y., Haesler, S., Vong, L., Lowell, B. B., and Uchida, N. (2012). Neurontype-specific signals for reward and punishment in the ventral tegmental area. Nature 482, 85-88. doi: 10.1038/nature10754

Cragg, S. J., and Rice, M. E. (2004). DAncing past the DAT at a DA synapse. Trends Neurosci. 27, 270-277. doi: 10.1016/j.tins.2004.03.011

Cruz, H. G., Ivanova, T., Lunn, M.-L., Stoffel, M., Slesinger, P. A., and Lüscher, C. (2004). Bi-directional effects of GABA(B) receptor agonists on the mesolimbic dopamine system. Nat. Neurosci. 7, 153-159. doi: 10.1038/nn1181

Dobi, A., Margolis, E. B., Wang, H.-L., Harvey, B. K., and Morales, M. (2010). Glutamatergic and nonglutamatergic neurons of the ventral tegmental area establish local synaptic contacts with dopaminergic and nondopaminergic neurons. $J$. Neurosci. 30, 218-229. doi: 10.1523/JNEUROSCI.3884-09.2010

Fields, H. L., Hjelmstad, G. O., Margolis, E. B., and Nicola, S. M. (2007). Ventral tegmental area neurons in learned appetitive behavior and positive reinforcement. Annu. Rev. Neurosci. 30, 289-316. doi: 10.1146/annurev.neuro. 30.051606.094341

Heikkinen, A. E., Möykkynen, T. P., and Korpi, E. R. (2009). Long-lasting modulation of glutamatergic transmission in VTA dopamine neurons after a single dose of benzodiazepine agonists. Neuropsychopharmacology 34, 290-298. doi: 10 . 1038/npp.2008.89

Hong, S., Jhou, T. C., Smith, M., Saleem, K. S., and Hikosaka, O. (2011). Negative reward signals from the lateral habenula to dopamine neurons are mediated by rostromedial tegmental nucleus in primates. J. Neurosci. 31, 11457-11471. doi: 10.1523/JNEUROSCI.1384-11.2011

Howe, M. W., Tierney, P. L., Sandberg, S. G., Phillips, P. E. M., and Graybiel, A. M. (2013). Prolonged dopamine signalling in striatum signals proximity and value of distant rewards. Nature 500, 575-579. doi: 10.1038/nature 12475

Ishikawa, M., Otaka, M., Neumann, P. A., Wang, Z., Cook, J. M., Schlüter, O. M., et al. (2013). Exposure to cocaine regulates inhibitory synaptic transmission from the ventral tegmental area to the nucleus accumbens. J. Physiol. 591, 48274841. doi: 10.1113/jphysiol.2013.262915

Jennings, J. H., Sparta, D. R., Stamatakis, A. M., Ung, R. L., Pleil, K. E., Kash, T. L., et al. (2013). Distinct extended amygdala circuits for divergent motivational states. Nature 496, 224-228. doi: 10.1038/nature12041

Johnson, S. W., and North, R. A. (1992a). Opioids excite dopamine neurons by hyperpolarization of local interneurons. J. Neurosci. 12, 483-488.

Johnson, S. W., and North, R. A. (1992b). Two types of neurone in the rat ventral tegmental area and their synaptic inputs. J. Physiol. 450, 455-468.

Joshua, M., Adler, A., Mitelman, R., Vaadia, E., and Bergman, H. (2008). Midbrain dopaminergic neurons and striatal cholinergic interneurons encode the difference between reward and aversive events at different epochs of probabilistic classical conditioning trials. J. Neurosci. 28, 11673-11684. doi: 10. 1523/JNEUROSCI.3839-08.2008

Lüscher, C., and Malenka, R. C. (2011). Drug-evoked synaptic plasticity in addiction: from molecular changes to circuit remodeling. Neuron 69, 650-663. doi: 10.1016/j.neuron.2011.01.017

Lüscher, C., and Ungless, M. A. (2006). The mechanistic classification of addictive drugs. PLoS Med. 3:e437. doi: 10.1371/journal.pmed.0030437

Margolis, E. B., Lock, H., Chefer, V. I., Shippenberg, T. S., Hjelmstad, G. O., and Fields, H. L. (2006). Kappa opioids selectively control dopaminergic neurons projecting to the prefrontal cortex. Proc. Natl. Acad. Sci. U S A 103, 2938-2942. doi: $10.1073 /$ pnas.0511159103

Matsumoto, M., and Hikosaka, O. (2009). Two types of dopamine neuron distinctly convey positive and negative motivational signals. Nature 459, 837-841. doi: 10 . 1038/nature08028

Mileykovskiy, B., and Morales, M. (2011). Duration of inhibition of ventral tegmental area dopamine neurons encodes a level of conditioned fear. J. Neurosci. 31, 7471-7476. doi: 10.1523/JNEUROSCI.5731-10.2011

Mirenowicz, J., and Schultz, W. (1996). Preferential activation of midbrain dopamine neurons by appetitive rather than aversive stimuli. Nature 379, 449451. Available at: http://www.nature.com/doifinder/10.1038/379449a0

Moss, J., and Bolam, J. P. (2008). A dopaminergic axon lattice in the striatum and its relationship with cortical and thalamic terminals. J. Neurosci. 28, 11221-11230. doi: 10.1523/JNEUROSCI.2780-08.2008

Oleson, E. B., and Cheer, J. F. (2012). A brain on cannabinoids: the role of dopamine release in reward seeking. Cold Spring Harb. Perspect. Med. 2:a012229. doi: 10. 1101/cshperspect.a012229

Olesson, E. B., and Cheer, J. F. (2013). On the role of subsecond dopamine release in conditioned avoidance. Front. Neurosci. 7:96. doi: 10.3389/fnins.2013.00096

Oleson, E. B., Gentry, R. N., Chioma, V. C., and Cheer, J. F. (2012). Subsecond dopamine release in the nucleus accumbens predicts conditioned punishment and its successful avoidance. J. Neurosci. 32, 14804-14808. doi: 10. 1523/JNEUROSCI.3087-12.2012

Overton, P., and Clark, D. (1992). Iontophoretically administered drugs acting at the $\mathrm{N}$-methyl-D-aspartate receptor modulate burst firing in A9 dopamine neurons in the rat. Synapse 10, 131-140. doi: 10.1002/syn.89010 0208

Peciña, S., and Berridge, K. C. (2013). Dopamine or opioid stimulation of nucleus accumbens similarly amplify cue-triggered "wanting" for reward: entire core and medial shell mapped as substrates for PIT enhancement. Eur. J. Neurosci. 37, 1529-1540. doi: 10.1111/ejn.12174

Pickel, V. M., Garzón, M., and Mengual, E. (2002). Electron microscopic immunolabeling of transporters and receptors identifies transmitter-specific functional 
sites envisioned in Cajal's neuron. Prog. Brain Res. 136, 145-155. doi: 10. 1016/s0079-6123(02)36014-x

Rudolph, U., Crestani, F., Benke, D., Brünig, I., Benson, J. A., Fritschy, J. M., et al. (1999). Benzodiazepine actions mediated by specific gammaaminobutyric acid(A) receptor subtypes. Nature 401, 796-800. doi: 10.1038/ 44579

Schultz, W. (2006). Behavioral theories and the neurophysiology of reward. Annu. Rev. Psychol. 57, 87-115. doi: 10.1146/annurev.psych.56.091103.070229

Schultz, W. (2013). Updating dopamine reward signals. Curr. Opin. Neurobiol. 23, 229-238. doi: 10.1016/j.conb.2012.11.012

Schultz, W., Apicella, P., and Ljungberg, T. (1993). Responses of monkey dopamine neurons to reward and conditioned stimuli during successive steps of learning a delayed response task. J. Neurosci. 13, 900-913.

Schultz, W., Dayan, P., and Montague, P. R. (1997). A neural substrate of prediction and reward. Science 275, 1593-1599. doi: 10.1126/science.275.5306.1593

Smith, K. S., Berridge, K. C., and Aldridge, J. W. (2011). Disentangling pleasure from incentive salience and learning signals in brain reward circuitry. Proc. Natl. Acad. Sci. U S A 108, E255-E264. doi: 10.1073/pnas.1101920108

Surmeier, D. J., Ding, J., Day, M., Wang, Z., and Shen, W. (2007). D1 and D2 dopamine-receptor modulation of striatal glutamatergic signaling in striatal medium spiny neurons. Trends Neurosci. 30, 228-235. doi: 10.1016/j.tins.2007. 03.008

Tan, K. R., Brown, M., Labouèbe, G., Yvon, C., Creton, C., Fritschy, J.-M., et al. (2010). Neural bases for addictive properties of benzodiazepines. Nature 463 769-774. doi: 10.1038/nature08758

Tan, K. R., Rudolph, U., and Lüscher, C. (2011). Hooked on benzodiazepines: GABAA receptor subtypes and addiction. Trends Neurosci. 34, 188-197. doi: 10. 1016/j.tins.2011.01.004

Tan, K. R., Yvon, C., Turiault, M., Mirzabekov, J. J., Doehner, J., Labouèbe, G., et al. (2012). GABA neurons of the VTA drive conditioned place aversion. Neuron 73 , 1173-1183. doi: 10.1016/j.neuron.2012.02.015

Ungless, M. A., Argilli, E., and Bonci, A. (2010). Effects of stress and aversion on dopamine neurons: implications for addiction. Neurosci. Biobehav. Rev. 35, 151156. doi: 10.1016/j.neubiorev.2010.04.006
Ungless, M. A., Magill, P. J., and Bolam, J. P. (2004). Uniform inhibition of dopamine neurons in the ventral tegmental area by aversive stimuli. Science 303, 2040-2042. doi: 10.1126/science. 1093360

Ungless, M. A., Whistler, J. L., Malenka, R. C., and Bonci, A. (2001). Single cocaine exposure in vivo induces long-term potentiation in dopamine neurons. Nature 411, 583-587. doi: 10.1038/35079077

van Zessen, R., Phillips, J. L., Budygin, E. A., and Stuber, G. D. (2012). Activation of VTA GABA neurons disrupts reward consumption. Neuron 73, 1184-1194. doi: 10.1016/j.neuron.2012.02.016

Xia, Y., Driscoll, J. R., Wilbrecht, L., Margolis, E. B., Fields, H. L., and Hjelmstad, G. O. (2011). Nucleus accumbens medium spiny neurons target nondopaminergic neurons in the ventral tegmental area. J. Neurosci. 31, 7811-7816. doi: 10.1523/jneurosci.1504-11.2011

Zweifel, L. S., Fadok, J. P., Argilli, E., Garelick, M. G., Jones, G. L., Dickerson, T. M. K., et al. (2011). Activation of dopamine neurons is critical for aversive conditioning and prevention of generalized anxiety. Nat. Neurosci. 14, 620-626. doi: $10.1038 / \mathrm{nn} .2808$

Conflict of Interest Statement: The authors declare that the research was conducted in the absence of any commercial or financial relationships that could be construed as a potential conflict of interest.

Received: 29 November 2013; accepted: 06 January 2014; published online: 22 January 2014

Citation: Creed MC, Ntamati NR and Tan KR (2014) VTA GABA neurons modulate specific learning behaviors through the control of dopamine and cholinergic systems. Front. Behav. Neurosci. 8:8. doi: 10.3389/fnbeh.2014.00008

This article was submitted to the journal Frontiers in Behavioral Neuroscience.

Copyright (c) 2014 Creed, Ntamati and Tan. This is an open-access article distributed under the terms of the Creative Commons Attribution License (CC BY). The use, distribution or reproduction in other forums is permitted, provided the original author(s) or licensor are credited and that the original publication in this journal is cited, in accordance with accepted academic practice. No use, distribution or reproduction is permitted which does not comply with these terms. 